Received: 16 March 2017

Accepted: 26 June 2017

Published online: 11 August 2017

\section{G protein stoichiometry dictates biased agonism through distinct receptor-G protein partitioning}

Lauriane Onfroy ${ }^{1}$, Ségolène Galandrin ${ }^{1}$, Stéphanie M. Pontier ${ }^{2}$, Marie-Hélène Seguelas ${ }^{1}$, Du N'Guyen ${ }^{1}$, Jean-Michel Sénard ${ }^{1,3} \&$ Céline Galés $^{1}$

Biased agonism at $\mathrm{G}$ protein coupled receptors emerges as an opportunity for development of drugs with enhanced benefit/risk balance making biased ligand identification a priority. However, ligand biased signature, classically inferred from ligand activity across multiple pathways, displays high variability in recombinant systems. Functional assays usually necessity receptor/effector overexpression that should be controlled among assays to allow comparison but this calibration currently fails. Herein, we demonstrate that $\mathrm{G} \alpha$ expression level dictates the biased profiling of agonists and, to a lesser extent of $\beta$-blockers, in a $\mathrm{G} \alpha$ isoform- and receptor-specific way, depending on specific $G$ protein activity in different membrane territories. These results have major therapeutic implications since they suggest that the ligand bias phenotype is not necessarily maintained in pathological cell background characterized by fluctuations in $\mathrm{G}$ protein expression. Thus, we recommend implementation of $\mathrm{G}$ protein stoichiometry as a new parameter in biased ligand screening programs.

G protein-coupled receptors (GPCRs) represent primary targets for drug development. However, many of these drugs display adverse side effects that can temper their clinical use. In recent years the concept of biased agonism has emerged and offers the possibility of alleviating and having more accurate control of this problem. Indeed, this now well-accepted paradigm defines the capability of a ligand to stabilize different conformations of a receptor and thus to activate or block a specific subset of intracellular associated signaling pathways.

This multitude of ligand-specific effects from a single receptor, also known as ligand texture, paves the way for the development of pathway-selective drugs with increased efficacy and less adverse effects ${ }^{1}$. This concept has been recently taken into consideration by pharmaceutical companies with several clinical trials based on the use of $\mathrm{G}$ protein- or $\beta$-arrestin-biased ligands currently in the pipeline ${ }^{2}$. Despite biased-agonism is more often contemplated from a clinical benefit standpoint, it could however as well contribute to an adverse benefit/risk balance in response to some drugs. Thus, $\beta$-blockers, originally developed to uniformly antagonize $\beta$-adrenergic receptors and currently considered as first-line drug in heart failure, have only recently been identified as biased ligands ${ }^{3}$ which could explain, in part, disparities in clinical efficacy among $\beta$-blockers ${ }^{4-6}$. Interestingly, the pharmacological imprinting of a biased ligand seems to be not only a fixed attribute of the chemical molecule but depends also on the cellular conditions. For instance, one study reported that classical antipsychotic drugs commonly antagonize the $\mathrm{D} 2-\mathrm{R} / \beta$-arrestin pathway ${ }^{7}$ which could refer to their efficacy, while, conversely, another demonstrated partial agonism in a similar cell system ${ }^{8}$. Likewise, the angiotensin II-mimicking peptide SII was originally described as a $\beta$-arrestin-biased AT1-R agonist with no activity on $\mathrm{G}$ protein signaling ${ }^{9}$ but afterwards was shown to exhibit partial agonist efficacy on the G protein as well ${ }^{10}$. Thus, biased agonism remains a fickle and still poorly comprehended phenomenon, the underlying molecular basis of which needs further exploration.

Until now, the pharmacological definition of biased agonists has been assigned to their effects on stabilizing of different receptor conformations ${ }^{11}$. Recently, temporal control of the different signaling pathways also appeared as an important determinant in differently modulating the biased-profiling of a ligand ${ }^{12,13}$. From a molecular standpoint, biased agonism redefines the pharmacological classification of ligand efficacy such that it no longer relies on a ligand/receptor bipartite but a more intricate ligand/receptor/effector tripartite, highlighting the importance

${ }^{1}$ Institut des Maladies Métaboliques et Cardiovasculaires, Institut National de la Santé et de la Recherche Médicale, U1048, Université de Toulouse, F-31432, Toulouse, France. ${ }^{2}$ Independent Researcher Ottawa, Ottawa, ON, Canada. ${ }^{3}$ Service de Pharmacologie Clinique, Centre Hospitalier Universitaire de Toulouse, Faculté de Médecine, Université de Toulouse, F-31000, Toulouse, France. Correspondence and requests for materials should be addressed to C.G. (email: celine.gales@inserm.fr) 


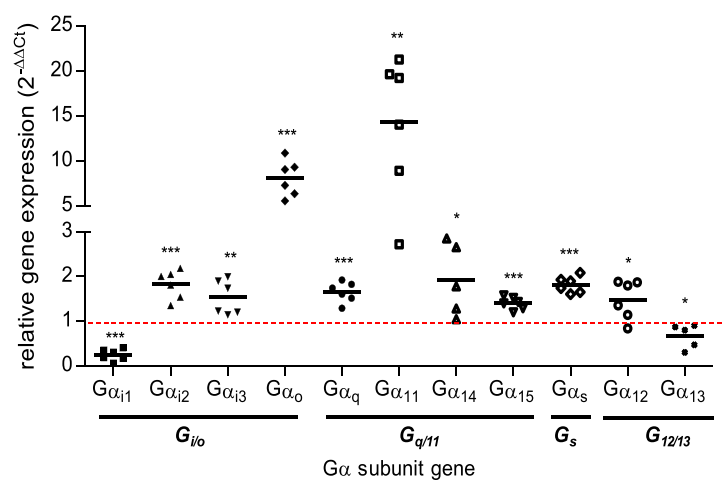

Figure 1. Quantification of heterotrimeric $G$ protein $\alpha$ subunits gene expression levels in hypertrophic cardiomyocytes. Comparative analysis of $G \alpha$ subunit gene expression of the four $G$ protein families $\left(G_{i / 0}, G_{q / 11}\right.$, $G_{s}, G_{12 / 13}$ ) in total RNA extracts from cardiomyocytes isolated from mice exhibiting or not (control) transversal aortic banding (TAC) obtained by Fluidigm Real-time qPCR. Results are expressed as the relative induction of the genes in $\mathrm{C} 57 \mathrm{Bl} / 6$ mice submitted to TAC for 15 days $(n=6)$ compared to control mice $(n=6)$ wherein genes expression are equal to 1 . The statistical significance of the change in expression level was assessed using unpaired Student's t-test. $* P<0.05, * * P<0.01, * * * P<0.001$

of the nature of the effector. In classical GPCR pharmacology, the influence of the relative receptor-G protein effector stoichiometry on ligand efficacy has been previously underlined ${ }^{14-18}$, however, this notion has surprisingly never been evaluated in terms of delineating the biased activity of ligands. On the contrary, evaluation of ligand biased activity in recombinant systems generally relies on the comparison of different signaling pathways, each of which are probed with different levels of receptor or effector without calibration (i.e. II messenger production in cells stably expressing a receptor versus $\beta$-arrestin recruitment to a transiently expressed receptor) which should preclude any comparison and biased activity quantification ${ }^{19}$. This could in part account for the discrepancies in biased activity described for some ligands. Moreover, abnormal stoichiometry of the receptor-effector system has long been viewed as a specificity of recombinant cell systems allowing higher expression levels compared to natural ones ${ }^{14}$. However, quantification of receptors or effectors in natural systems has mostly relied on studies using tissues that express different cell types, thus preventing accurate and specific quantification for each cell type. In addition, physiological receptor-effector stoichiometry is not static, for instance, Gpr176 is expressed in a circadian manner by suprachiasmatic nucleus neurons ${ }^{20}$. Finally, both receptor and effector expression also undergo modifications in pathological contexts. Thus, G protein ${ }^{21}$ as well as GPCR expression profiles ${ }^{22}$ are profoundly modified during immune cell maturation. Some studies have also already described the downregulation of receptors, $G$ proteins and secondary effectors in heart failure ${ }^{23-25}$, while other alterations in G protein expression have been reported in cancer ${ }^{26}$ and Parkinson's disease ${ }^{27}$. This pathological cell environment should be better considered from a clinical biased-drug perspective and has rarely been systematically considered for in vitro drug screening.

In this study, we took advantage of HEK293T recombinant cell system that allows high flexibility in protein expression to perform the first systematic calibrated study evaluating and accurately quantifying the effect of varying the expression levels different heterotrimeric G proteins, the common effectors of all GPCR families, on the efficacy of GPCR ligands on G protein activation. We show that the G $\alpha$ subunit expression levels plays a prominent role in the biased profiling of $\beta$-agonists but also antagonists, affecting both their potency and/or efficacy by targeting different membrane distribution of receptor- $G$ protein populations. More surprisingly, we found that, in the resting state, the level of $\mathrm{G} \alpha$ expression influences the partitioning of not only $\mathrm{G} \alpha$ but also the co-expressed receptor in different cholesterol-enriched membrane domains. Thus, our data provide critical insights toward quantification of biased agonism but also improve our knowledge on the $\mathrm{G}$ protein/receptor interplay at resting state.

\section{Results}

Alterations of $\mathrm{G} \alpha$ subunits gene expression in failing cardiomyocytes. We first wondered whether

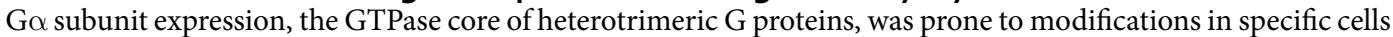
from pathological tissue. We thus evaluated the relative expression of the major $\mathrm{G} \alpha$ subunits from all $\mathrm{G}$ protein

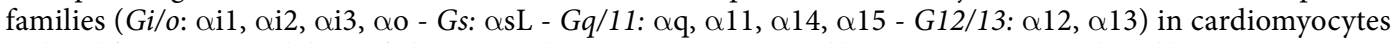
isolated from mice with heart failure secondary to an experimental barometric stress induced by transverse aortic constriction previously described ${ }^{28}$. Quantitative PCR led to the identification of important modifications

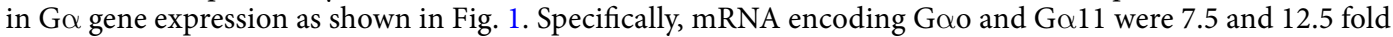
over-expressed respectively in failing cardiomyocytes compared to control cardiomyocytes. All other Go subunit mRNAs were also prone to a significant but less substantial up-regulation oscillating between 1.5 and 2 fold, with

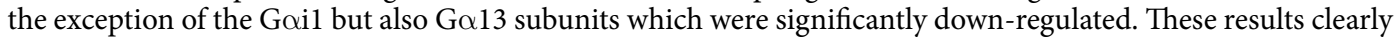
indicate that the gene expression of the $\mathrm{G} \alpha$ subunits is highly prone to important variation in diseased cardiomyocytes, most likely suggesting correlated modifications in the resultant $\mathrm{G} \alpha$ proteins. Based on these results, we then decided to explore the relationship between ligand pharmacology and G protein stoichiometry more 


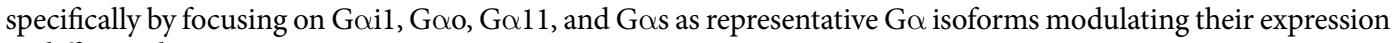
to different degrees.

Go stoichiometry dictates the maximal efficacies of different ligands. We first performed a preliminary screen in recombinant HEK293T cells to test the consequences of variable G $\alpha$ subunits expression on their activation. Gos and GooA activation was measured following synthetic isoproterenol (ISO)-stimulation

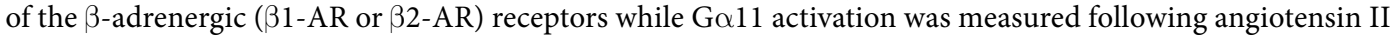
(AngII)- stimulation of the angiotensin II (AngII) type 1 receptor (AT1-R). These GPCRs are all prominent drug targets in heart failure. Direct activation of the specific $\mathrm{G \alpha}$ subunits was assessed using BRET ${ }^{2}$ probes which we previously described ${ }^{10,29}$ by measuring the interaction between the specific G $\alpha$-Rluc8 and GFP10-G $\gamma 2$ subunits in the presence of the complementary untagged G $\beta 3$ which is specifically expressed in adult cardiomyocytes ${ }^{30}$. Indeed, these $\mathrm{G}$ protein activity-BRET probes provide powerful and accurate tools to delineate ligand pharmacology. Thus, we have previously shown that they can not only allow sense the agonist efficacy of ligands, reflected by a decrease in the BRET signal ${ }^{10,29,31}$ (negative BRET modulation), but conversely can also detect inverse ago-

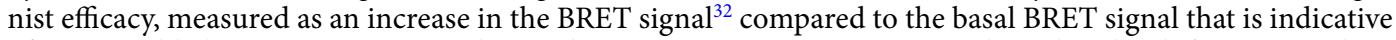
of preassembled inactive G $\alpha \beta \gamma$ complexes. Thus, $\mathrm{G}$ protein activation was evaluated under different $\mathrm{G} \alpha$-Rluc 8 expression levels that were systematically quantified by measuring the total amount of luminescence. In these experiments, we used receptor excess and saturating ligand concentration to ensure depicting of the maximal ligand/receptor/G protein complexed fractions of the receptor population. Reference agonist ligands (i.e. ISO or

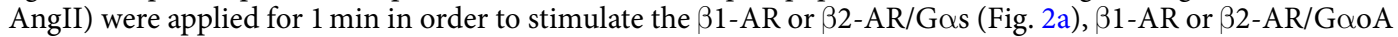

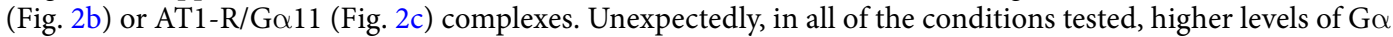
subunit expression correlated with reduced ligand-induced negative BRET modulation (Fig. 2). These results are particularly unexpected in a model that classically predicts an increased in receptor-G protein coupling when $G$ proteins are in excess. Nevertheless, they support the idea that high G protein expression levels could affect the pharmacological profile and the efficacy of a given ligand.

Go isoform and stoichiometry dictate varying $\beta$-adrenergic ligand pharmacology. To gain further insight into the influence of Go expression level on ligand efficacy, we decided to focus on the $\beta 1$ - and $\beta 2$-AR

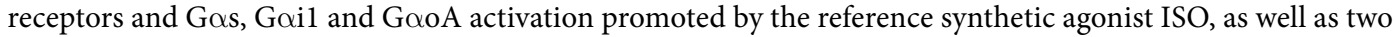
natural agonists of these GPCRs, epinephrine (EPI) and norepinephrine (NE).

We first measured the activation of these different $G$ protein activity-BRET biosensors in HEK293T cells in the absence of $\beta 1$ - and $\beta 2$-AR over-expression to evaluate the potential contribution of endogenous adrenergic responses (Supplementary Fig. 1). Importantly, G protein activation was evaluated with both high and low G $\alpha$ expression levels that were tightly calibrated to similar levels in each transfection for all subunits (Supplementary Fig. 1, inset). It is also worth noting that, while the various Go-Rluc8 subunits used in these experiments were over-expressed at two distinct expression levels, the overall expression of these recombinant proteins was still below the expression level of the corresponding endogenous G $\alpha$ subunit, as shown by western-blotting in the case of the Gos subunit (Supplementary Fig. 2). As expected, although we did not detect any significant Gos activation, except in the presence of EPI at high Gos expression levels (Supplementary Fig. 1a), EPI promoted a significant activation of both Goil and GooA whereas NE more selectively activated GooA and ISO was ineffective (Supplementary Fig. 1b,c), thus confirming the presence of endogenous adrenergic receptors as known in HEK293T cells. However, the endogenous receptors were not problematic when both $\mathrm{G}$ protein biosensors and receptors were over-expressed, since under these experimental conditions, the $G$ protein activation response was always shifted toward the over-expressed receptor $(R)-G$ protein $(G)$ complex. Indeed, when we sensed $G$ protein activation in the presence or absence of $\beta 1-/ \beta 2$-AR (Supplementary Fig. 3a) or conformational rearrangements within the different R-G complexes (Supplementary Fig. 3b) by measuring BRET between G $\alpha$-Rluc 8 and $\beta 1$-AR-GFP10 or $\beta 2$-AR-GFP10 as previously described ${ }^{10,29}$, we found a good correlation between maximal agonist responses in R-G conformational changes and $\mathrm{G}$ protein activation profiles obtained with over-expressed $\beta 1$-AR or $\beta 2-A R$ (Supplementary Fig. 3c). In contrast, no parallel could be found between conformational changes stabilized by $\beta$-agonists on the Gai1/ $\beta 1$ - or $\beta 2$-AR complexes and G protein activation profiles obtained with endogenous adrenergic receptors (Supplementary Fig. 3d). Moreover, the maximal Gail activation response promoted by the different $\beta$-AR agonists in the presence of over-expressed $\beta 1$ - or $\beta 2$-AR agreed with the rank order selectivity of ligand potency for these receptors with EPI $>$ NE for $\beta 2$-AR while NE $>$ EPI for $\beta 1$-AR. These results were also corroborated by cAMP measurements (Supplementary Fig. 4) performed in the presence or not of G $\alpha$ s, $\beta 1$ - or $\beta 2$-AR over-expression under conditions similar to those of the BRET experiments that measured the $G$ protein activation. EPI and NE promoted significant concentration-dependent cAMP production with endogenous receptors but with a low potency that was always leftward shifted and was associated with marked increase in maximal efficacy when the $\beta 1$ - or $\beta 2$-AR were over-expressed (Supplementary Fig. 4; Supplementary Table 1). Further addition of the Gos subunit potentiated the maximal efficacy of EPI and NE independently of its expression level at $\beta 1$-AR (Supplementary Fig. $4 \mathrm{a}$ ) but not at $\beta 2$-AR (Supplementary Fig. $4 \mathrm{~b}$ ), indicative of a highly selective influence of $\mathrm{G} \alpha$ on receptor-mediated adenylyl cyclase activation.

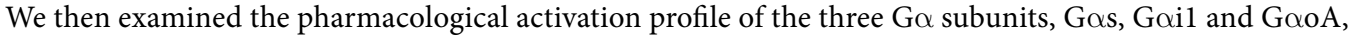
when expressed at high and low levels in the presence of $\beta 1$ - or $\beta 2$-AR (Fig. 3). In these experiments, each receptor was calibrated to ensure similar cell surface expression levels for each of the three Go subunits experimental condition (either high and low expression) but not between receptors (Supplementary Fig. 5), while all Go subunits expression levels (high or low) were similarly calibrated between the three isoforms (Supplementary Fig. 6). For Gos activation, both NE and EPI displayed different dose-response curves in the presence of $\beta 1$-AR depend-

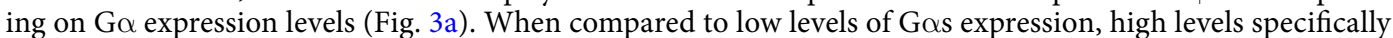
increased EPI potency, but decreased the maximal efficacy of NE (Supplementary Table 2). Interestingly, while the 
a

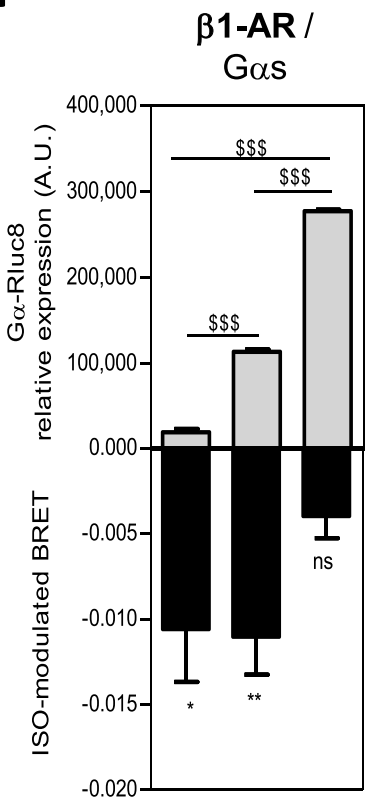

b

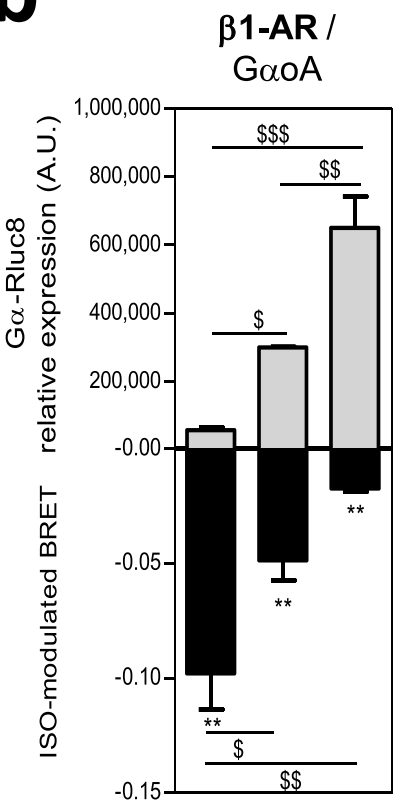

B2-AR /

Gas

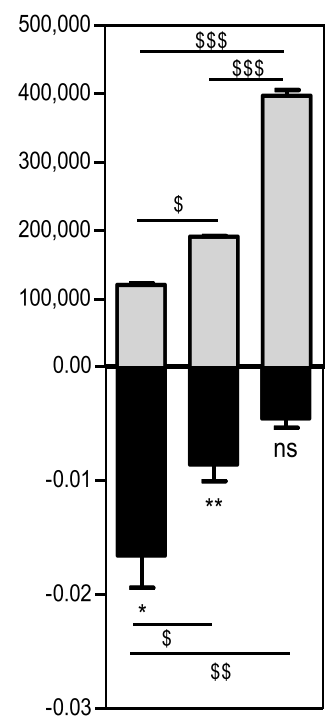

B2-AR / GoOA

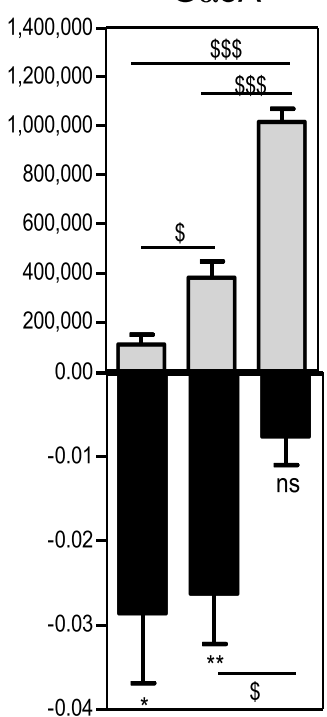

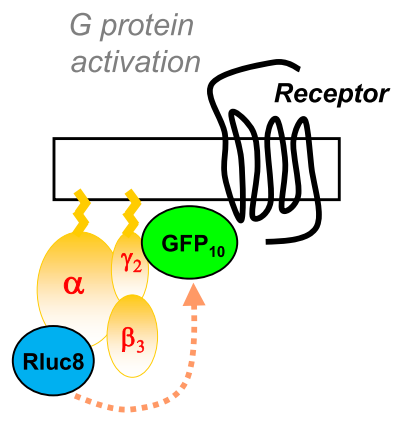

C

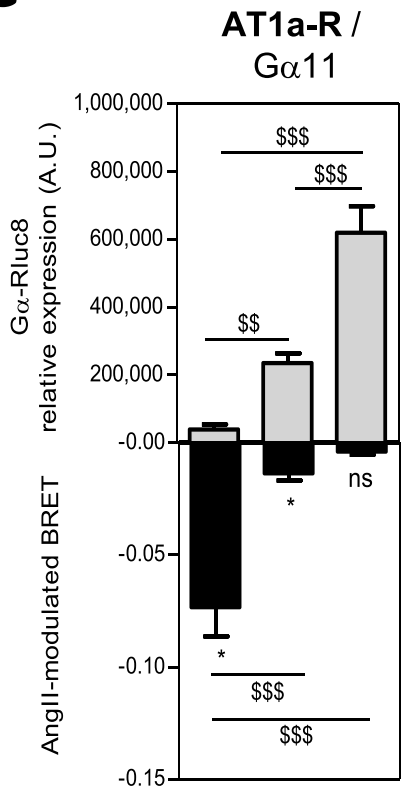

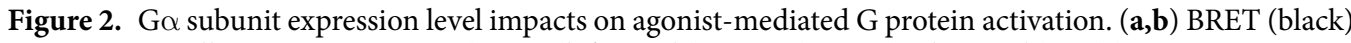
in HEK293T cells co-expressing HA- $\beta 1$-AR (left panels) or HA- $\beta 2-A R$ (right panels) or (c) AT1a-R receptors

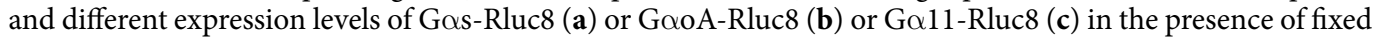
GFP10-G $\gamma 2$ and G $\beta 3$ untagged subunits. Cells were stimulated or not for $1 \mathrm{~min}$ with $10 \mu \mathrm{M}$ of the indicated selective agonists isoproterenol (ISO) or angiotensin II (AngII). Results are expressed as the difference in BRET signals measured in presence and absence of ligand. Data represent the mean \pm s.e.m. of at least three independent experiments. The statistical significance between stimulated and unstimulated cells was assessed using paired Student's t-test $(* P<0.05, * * P<0.01, * * * P<0.001)$. G $\alpha$-Rluc8 relative expression levels (gray) were depicted by the luminescence measurement and represent the mean \pm s.e.m. of at least three independent experiments. The statistical significance of the difference in luminescence between G $\alpha$ expression levels was assessed using one-way ANOVA followed by a Tukey's multiple comparison test $\left({ }^{\$} P<0.05,{ }^{\$ \$} P<0.01\right.$, $\left.{ }^{\$ \$} P<0.001\right)$. 
a
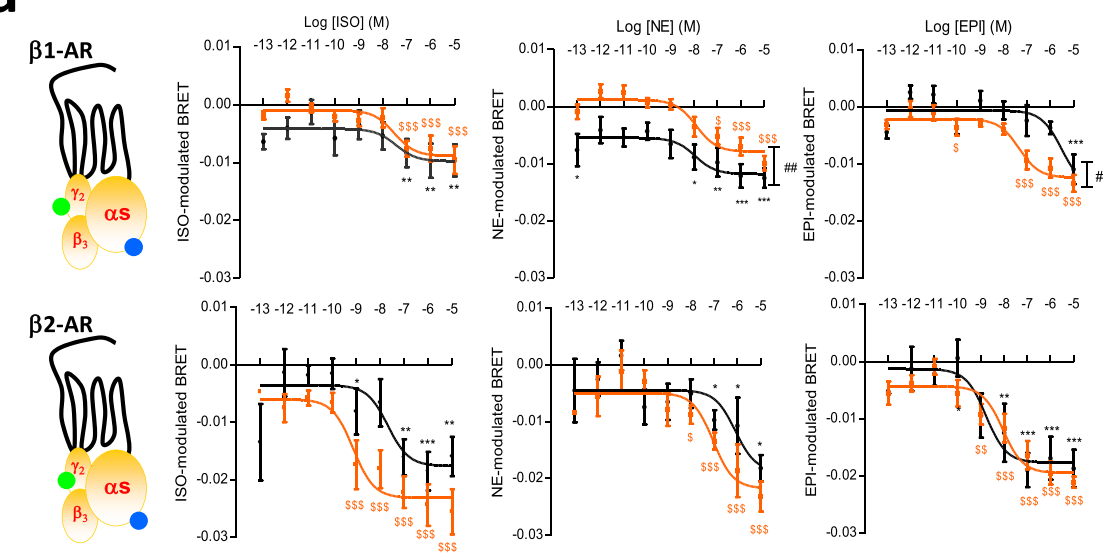

b
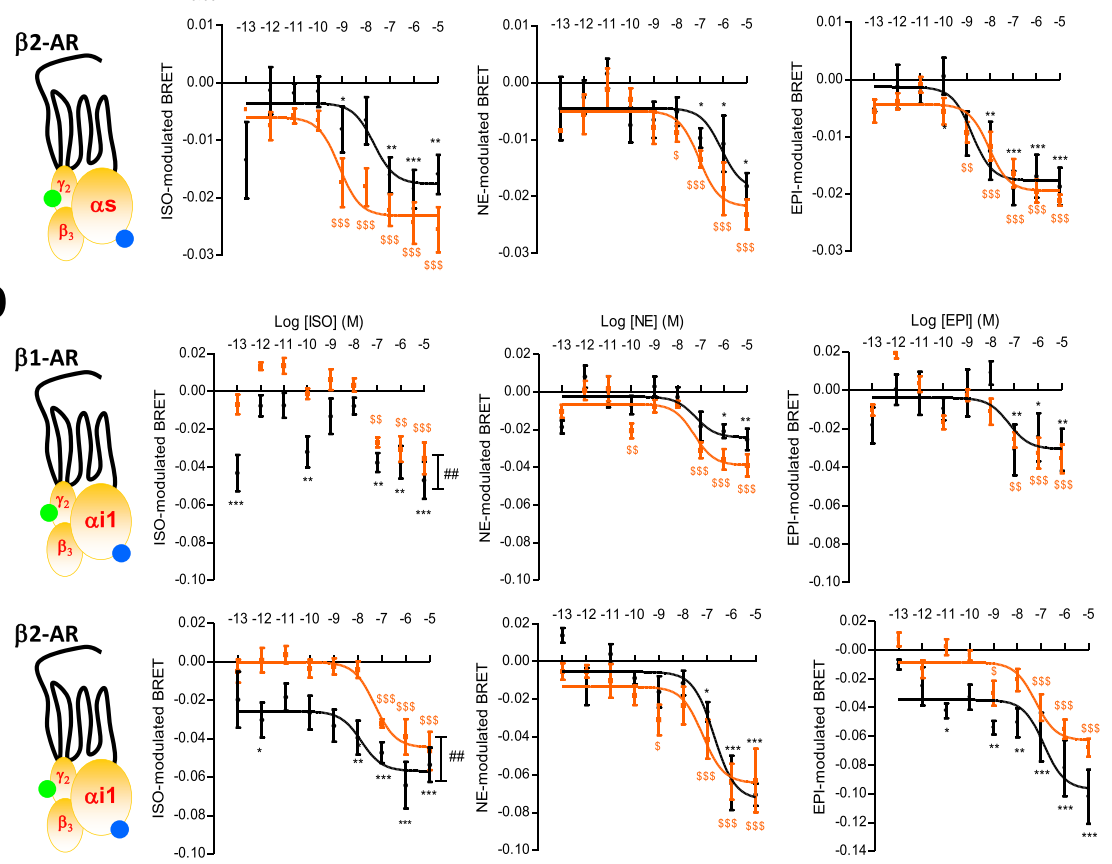

C
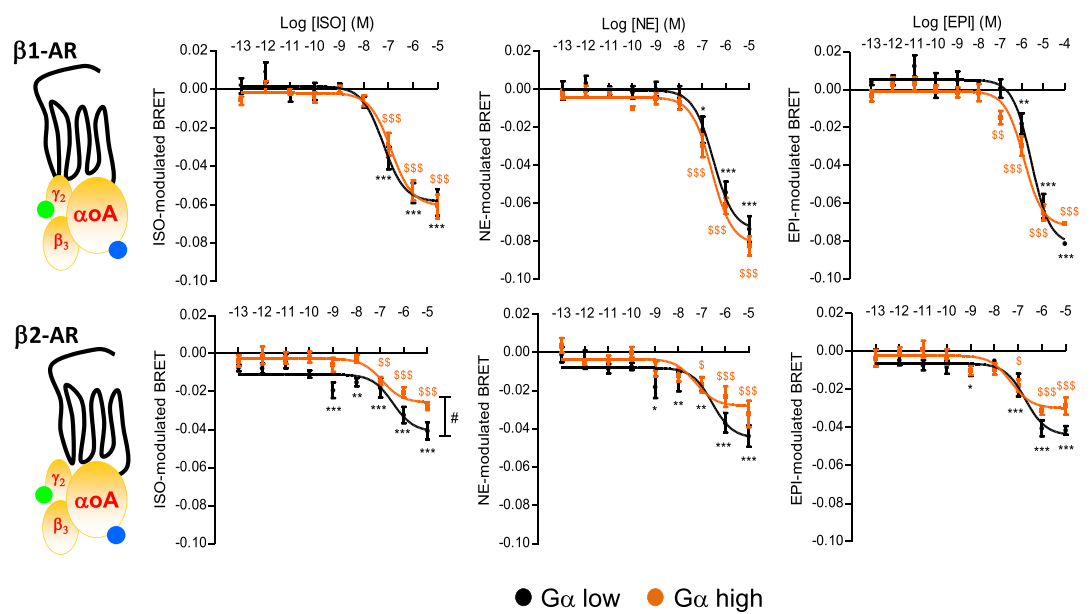

Figure 3. Influence of the G $\alpha$ subunit expression level on the concentration-response curves of $\beta$-AR agonistsmediated G protein activation. (a-c) BRET in HEK293T cells co-expressing HA- $\beta 1$-AR (upper panels) or HA-32-AR (lower panels) receptors and low (black) or high (orange) expression levels of Gos-Rluc8 (a), Goil-Rluc8 (b) or GooA-Rluc8 (c) in presence of fixed GFP10-G $\gamma 2$ and G $\beta 3$ untagged subunits. Cells were stimulated or not for $1 \mathrm{~min}$ with increasing concentrations of the indicated agonists (Isoproterenol, ISO; Norepinephrine, NE; Epinephrine, EPI). Results are expressed as the difference in BRET signals measured in presence and absence of ligand. Data represent the mean \pm s.e.m. of at least four independent experiments. The statistical significance between unstimulated cells and cells stimulated with the different ligand concentrations in low (*) and high (\$) Go-Rluc8 conditions was assessed using one-way ANOVA followed by a Dunnett's multiple comparison test $(* P<0.05, * * P<0.01, * * * P<0.001)$. The statistical significance between low and high dose-response curves was assessed using two-way ANOVA followed by a Bonferroni post-test $\left({ }^{\#} P<0.05\right.$, $\left.{ }^{\# \# P}<0.01\right)$. 
a

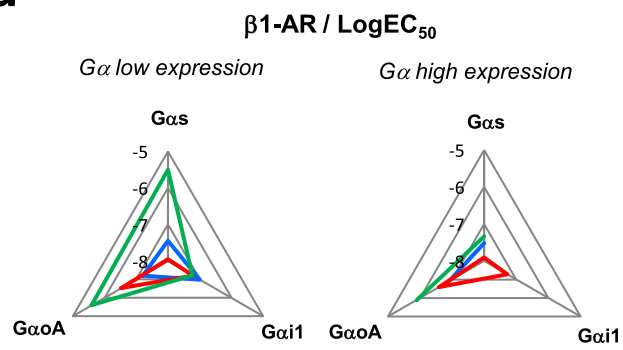

C

$\beta 1-A R / E m a x$

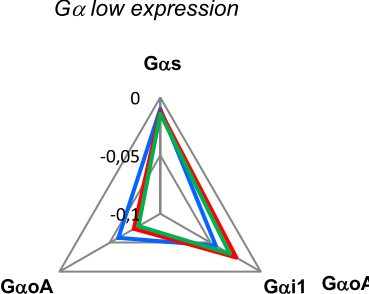

G $\alpha$ high expression

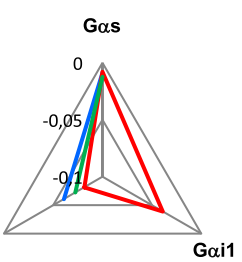

b

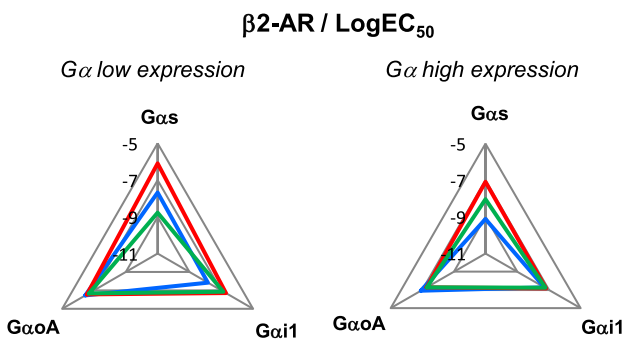

Figure 4. Schematic multi-axial profiling of $\beta$-AR agonist efficacies and potencies at $G$ protein activation with different $\mathrm{G} \alpha$ expression levels. (a-d) ISO, NE or EPI potencies $(\mathbf{a}, \mathbf{b})$ or maximal efficacies $(\mathbf{c}, \mathbf{d})$ at $\mathrm{G}$ protein (Gos, Goi1, GooA) activation upon stimulation of $\beta 1-A R(\mathbf{a}, \mathbf{c})$ or $\beta 2-A R(\mathbf{b}, \mathbf{d})$ presented in Fig. 3 and Supplementary Tables 2 and 3 are shown in a web.

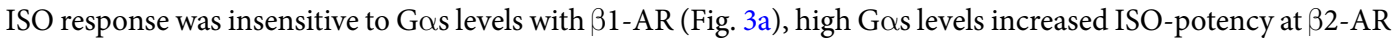
(Fig. 3a; Supplementary Table 3). For Goil activation by $\beta 1$-AR (Fig. 3b), ISO displayed an atypical pharmacological profile with a double-bell-like shaped dose-response curve showing significant activation at very low ligand concentrations in the presence of low Goil levels. On the contrary, the expression level of Goil had no significant impact on NE and EPI responses. In the presence of $\beta 2$-AR (Fig. 3b), whereas Gail activation was detected at low Goil levels in the low ISO and EPI concentration ranges and specifically potentiated the maximal activation promoted by these two ligands, Gail levels had no detectable influence on the NE response. Although GooA belongs to the same Gi/o family as Gail, the dose-response curve for each ligand was quite different (Fig. 3b,c). ISO, NE and EPI displayed similar concentration-response profiles with $\beta 1$-AR in the presence of either low or high GooA levels while for $\beta 2$-AR, low GooA levels increased the maximal efficacy of all three ligands (Fig. 3c). Interestingly, when similar G $\alpha$ stoichiometry experiments were conducted with some $\beta$-blockers used (bisoprolol and metoprolol) or not (timolol) in the treatment of patients with heart failure, results, despite more fluctuating, highlighted lesser susceptibility of this pharmacological class toward $\mathrm{G} \alpha$ level compared to $\beta$-agonists (Supplementary Fig. 7). When considering Gos, no significant activation was measured for none of the three ligands through $\beta 1$ - or $\beta 2-A R$, independently of the Gos level, in agreement with their antagonistic properties despite some agonist efficacy could be depicted at high timolol concentrations for high Gos expression. Agonist efficacy of $\beta$-blockers

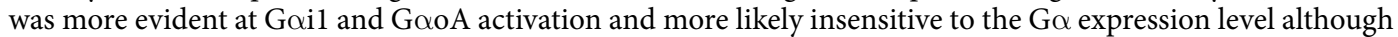
low levels of some $\mathrm{G} \alpha$ can exacerbate the agonist dose-response but with high variability (BISO/ $\beta 2-\mathrm{AR} / \mathrm{G} \alpha \mathrm{il}$; BISO//1-AR/GooA; METO/ $\beta 2-A R / G \alpha o A)$. The LogEC50 and Emax values obtained with the $\beta$-agonists have been graphically represented in a web format to better appreciate the fingerprint of each $\beta$-adrenergic agonist in activating the three $\mathrm{G} \alpha$ subunits at both high and low expression levels for $\beta 1$-AR and $\beta 2$-AR (Fig. 4). All of the webs clearly highlight that low versus high Go expression levels modified the bias fingerprint for almost all ligands and the two receptors, thus demonstrating that the expression level of the Go subunit accurately dictates bias of ligands. It is worth noting that all three $\beta$-adrenergic agonists displayed different fingerprints from each other for each web, most likely indicating that they behaved as biased ligands.

$\mathrm{G} \alpha$ isoform and stoichiometry dictates selective $\beta$-adrenergic agonist responses through different membrane R-G populations. The biased activity of ligands has been assigned to their ability to stabilize distinct GPCR conformations ${ }^{11}$. Moreover, membrane lipids have also recently been shown to play a key role in the allosteric modulation of GPCR activity by stabilizing specific receptor conformations ${ }^{33}$. Also, one mechanism which could account for the biased pharmacology of $\beta$-adrenergic agonists as a function of the

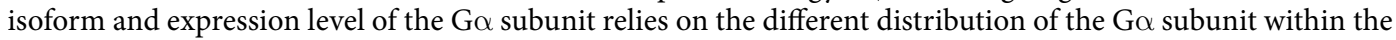
plasma membrane in diverse lipid territories that could interact with different receptor populations. Hence, one ligand could promote different conformations of $\beta$-AR/G protein complexes with different outputs depending on their compartmentalization at the cell surface. We first tested this hypothesis by decoding the conformations of 
a

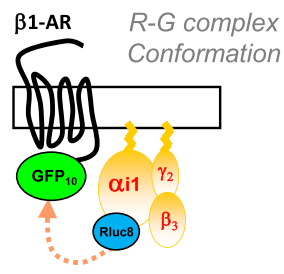

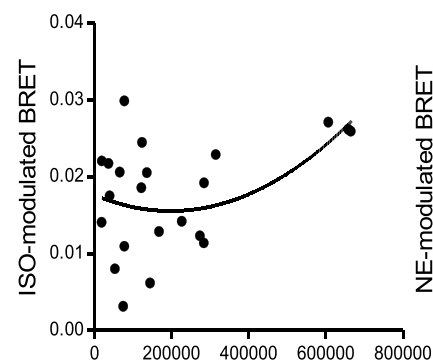

$\mathrm{G} \alpha$-Rluc8 relative expression (Luminescence A.U.)

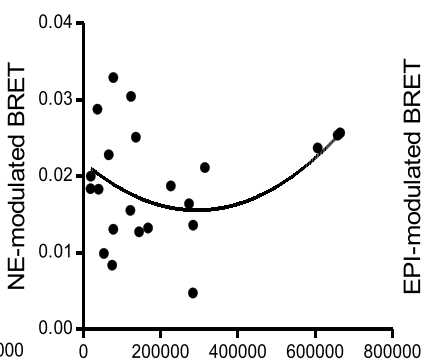

$\mathrm{G} \alpha-$ Rluc8 relative expression (Luminescence A.U.)

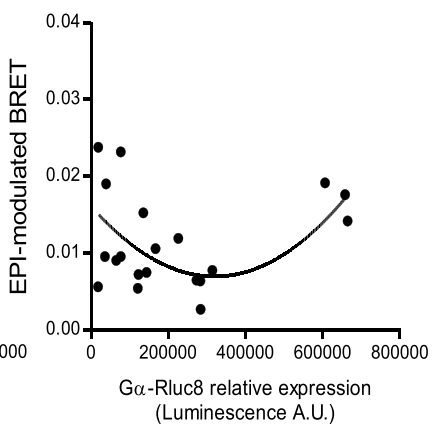

(Luminescence A.U.)

b
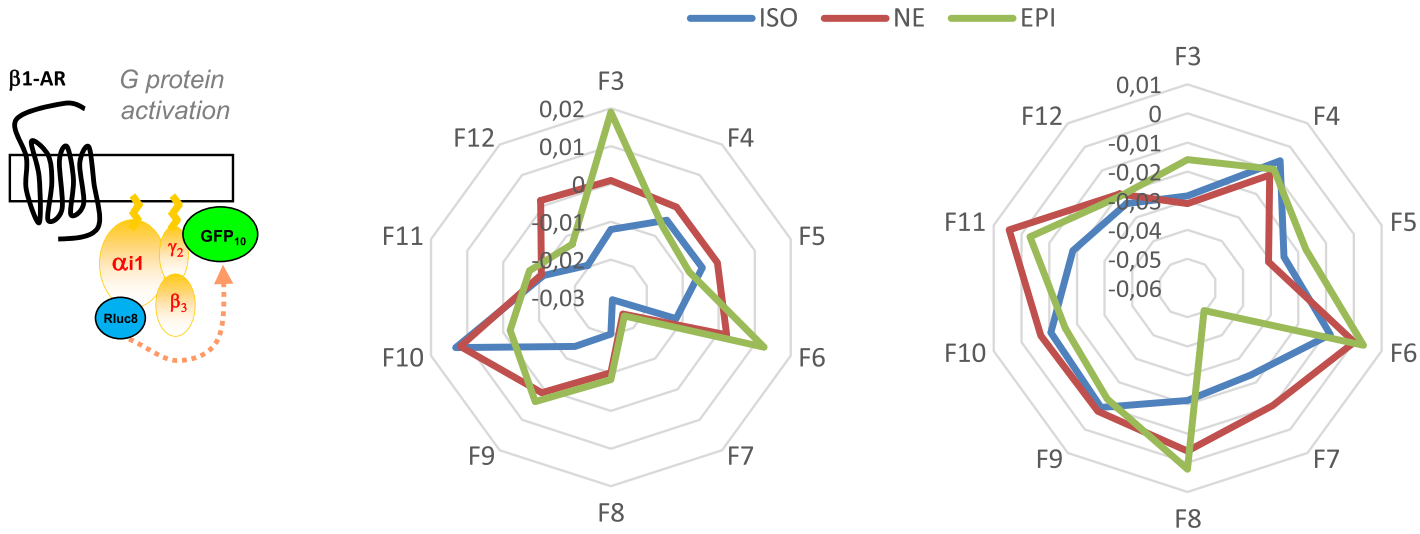

Gai1-Rluc8 relative expression

Figure 5. Influence of the $\mathrm{G} \alpha$ stoichiometry on $\beta$-adrenergic agonist responses through different membrane Receptor-G protein populations. (a) Receptor-G protein complexes conformations assessed by BRET in HEK293T cells co-expressing $\beta 1$ AR-GFP10 receptor and different expression levels of Gail-Rluc8 in presence of fixed $\mathrm{G} \gamma 2$ and $\mathrm{G} \beta 3$ untagged subunits. Cells were then stimulated or not for $1 \mathrm{~min}$ with $10 \mu \mathrm{M}$ of the indicated ligands (ISO, NE or EPI). Results are expressed as the difference in BRET signals measured in presence and absence of ligand and are representative of four independent experiments. Curves were generated using a polynomial quadratic model. (b) G protein activation measured in sucrose fractions by BRET in HEK293T cells, co-expressing HA- $\beta 1$ AR receptor and different expression levels of Goil-Rluc8 in presence of fixed GFP10-G $\gamma 2$ and GB3 untagged subunits, and processed for raft purification. Results are expressed as the difference in BRET signals measured in presence and absence of ligand and plotted in the wheel diagram from the different sucrose fractions (F3 to F12). They are representative of three independent experiments.

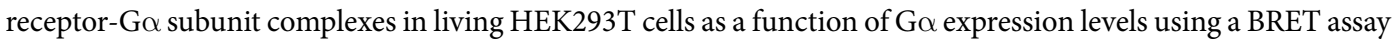

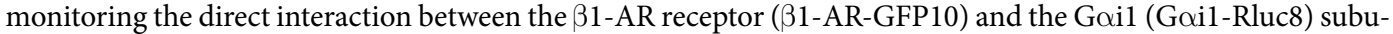
nit as previously described ${ }^{10,29}$. The R-G conformations stabilized by ISO, NE or EPI were strongly dependent on Goil expression (Fig. 5a). Indeed, the relationship between R-G conformations and Goil expression levels was better fitted using a second order polynomial curve model than a linear one for all of the ligands (F values: 1.775, 2.932 and 7.325 for ISO, NE and EPI respectively). The difference in curvilinearity between the three ligands further confirmed their biased efficacy relative to Go expression levels. Noteworthy, for all ligands tested, the number of R-G conformations increased considerably inversely to Go expression levels. These results highly suggest that the different $\beta$-AR agonists recognize distinct populations of receptor-G protein complexes at the cell surface, most likely located in different membrane territories. To gain further insight into this assumption, we directly measured $\mathrm{G}$ protein activation in different lipid membrane territories. More specifically, low density membrane microdomains ("rafts"), the most common ones, were isolated by cell solubilization with $1 \%$ Triton X-100 at $4{ }^{\circ} \mathrm{C}$ followed by sucrose gradient separation, as previously described ${ }^{34}$. Thus, we measured BRET ${ }^{1}$ on each sucrose fraction obtained from the solubilization of HEK293T cells co-expressing $\beta 1$-AR with high expression levels of Goil-RLuc8 in the presence of Venus-G $\gamma 2$ and G $\beta 3$ subunits, similarly to that in Fig. 3. G protein activation triggered by the three agonists ISO, NE and EPI, was plotted in a web as a function of the sucrose fraction (F3 to F12)

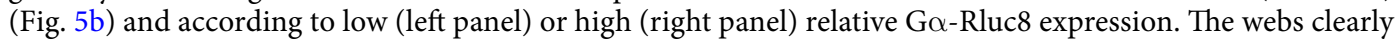


indicate that each agonist initiated a specific activation profile that was dependent not only on the fraction but

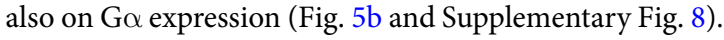

$\mathrm{G} \alpha$ isoform and stoichiometry dictates differential $\beta 2$-adrenergic receptor membrane partitioning. To gain further insight into the molecular mechanisms underlying such selectivity of $\beta$-adrenergic agonist responses among different membrane lipid territories depending on the $\mathrm{G} \alpha$ isoform and expression level,

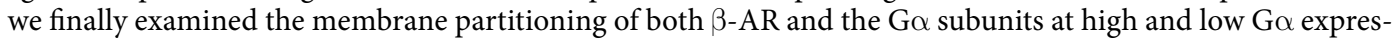
sion level conditions at resting state. HEK293T cells, co-expressing $\beta 1$ - or $32-\mathrm{AR}$ and Gos, Goil or GooA in the presence of $\mathrm{G} \gamma 2$ and $\mathrm{G} \beta 3$ subunits similarly to that in Fig. 3, were proceeded for raft purification and we then studied the distribution of both the $31 / 2-\mathrm{AR}$ and the $\mathrm{G} \alpha$ subunits at high or low $\mathrm{G} \alpha$ expression levels similar to the previous BRET experiments (Fig. 6). To improve detection sensitivity, we took advantage of $\beta 1 / 2$-AR-Rluc or $\mathrm{G \alpha}$-Rluc8 constructs whose luminescence measurements can more accurately quantify both proteins separately in each sucrose fraction. The GM1 raft marker was used as a positive control to identify raft-enriched fractions in dot-blot. Validation of luminescence recording as a valuable tool in depicting protein compartmentalization in lipid rafts isolated in a sucrose gradient, was provided by a GABAB-R2-Rluc fusion construct encoding the prototypical neuronal R2 subunit of the GABAB receptor which usually functions in these specific microdomains ${ }^{35}$. As expected, when overexpressed in HEK293T cells, GABAB-R2-Rluc was concentrated mostly in raft-enriched fractions (Fractions 2-6) at the top of the gradient but could also be detected in some non raft fractions at the extreme gradient bottom (Fractions 10-12) (Supplementary Fig. 9). When following Go subunit distribution (Fig. 6a), each of the three subunits exhibited a specific distribution pattern. In agreement with their lipid modifications $^{36}$, in the absence of receptor co-expression and independently of their expression levels, Goil and GooA were essentially concentrated in raft-enriched fractions while Gos was more largely distributed in non-raft domains. Interestingly, further addition of $\beta 2$-AR (Fig. 6a, lower panels) significantly modified the repartition pattern of some $\mathrm{G} \alpha$ isoforms at high expression levels, with the Goil subunit more shifted in the raft domains to the detriment of the non-rafts ones while conversely Gas expression was shifted to non-raft domains at the gradient bottom to the detriment of the rafts. Moreover, $\beta 2$-AR expression shifted the entire GooA profile to raft-concentrated domains at both high and low expression levels, decreasing GooA expression in non-rafts. This observation is receptor-specific as different $\mathrm{G} \alpha$ gradient profiles were obtained with $\beta 1$-AR (Fig. 6a, middle panels). Indeed, $\beta 1$-AR expression stretched the Gos repartition along the gradient, thus increasing its expression in both raft and non-raft domains for low and high Gos expression levels, whereas Goil had decreased expression in non-raft fractions in favor of rafts. 31 -AR differently impacted on GooA distribution according to its expression levels as it decreased GooA in non-rafts while increasing it in rafts when considering low GooA expression level. At high GooA expression level, the $\mathrm{G}$ protein is conversely increased in non-raft fractions.

When now considering the $\beta 2$-AR distribution, and as previously reported ${ }^{34}$, in the absence of $\mathrm{G}$ protein over-expression, 32 -AR was enriched in raft domains (Fig. 6b, upper panels). Unexpectedly, while the distribution profiling of 32 -AR was not modified by either high or low levels of GooA expression (Fig. 6b, lower panels), in the presence of Gas it shifted toward non-raft fractions. This could in part explain, together with the specific Gas distribution, the modifications in cAMP production when both the $\beta 2$-AR and the Gos proteins were over-expressed (Supplementary Fig. 4). In the absence of $G$ protein expression, $\beta 1$-AR repartition was consistently more concentrated in non-raft domains compared to 32 -AR (Fig. 6b, upper panels) and was less impacted by Gas or Gail expression (Fig. 6b, middle panels). However, high GooA expression increased $\beta 1$-AR expression in rafts and decreased it in non-rafts. Oddly, modification of the $\beta$-AR raft-profiling in the presence of the different $\mathrm{G} \alpha$ subunits did not necessarily correlate with the Go profiling and vice-versa, suggesting that the profiling of the receptor co-expressed with a peculiar $\mathrm{G} \alpha$ protein most likely results from a specific interplay between the two proteins and not from the specific influence of one protein on the other.

These result highlights for the first time the impact of the Go subunit on the receptor membrane distribution that could account for specific biased pharmacology.

\section{Discussion}

In this study, we used controlled experimental conditions with accurate calibration of protein expression in a recombinant cell system, and have identified the importance of the stoichiometry of the G protein G $\alpha$ subunit in the biased-activity of GPCR ligands. This occurs through an unexpected molecular mechanism at "basal state" of the cell governing different distribution of both the $\mathrm{G} \alpha$ subunit but also the receptor within specific membrane subdomains.

A major finding of our study is the influence of $\mathrm{G} \alpha$ subunit expression levels on both the potency and efficacy of agonist ligands at a particular receptor. It is now well accepted that a precise stoichiometry among the signaling components determines the predominant signaling response, and the influence of GPCR and G protein expression levels on ligand efficacy have been previously documented ${ }^{14,15}$. Most studies have indeed extensively examined receptor stoichiometry on ligand behavior but only few data are available regarding the impact of $G$ protein expression ${ }^{18}$. The influence of $\mathrm{G}$ protein over-expression has essentially been examined on inverse agonist efficacy at constitutive receptors and interpreted as a result of modulation of the R/R* ratio, in agreement with the predictions from the allosteric model for receptor activation (extended ternary/cubic model $)^{37,38}$. According to this classical model, ligand efficacy should evolve through a linear relationship with $\mathrm{G}$ protein expression levels, consistent with different degrees of this ligand to stabilize the $R$ or $R *$ receptor state as a function of the $G$ protein level. However, although this assumption can be easily inferred from reconstituted systems ${ }^{38}$, it clearly differs from our results since in our living cell system with constant and saturating receptor concentrations, low and high levels of $\mathrm{G \alpha}$ subunit over-expression were non linearly correlated with ligand potency and efficacy at $\mathrm{G}$ protein activation and instead dictated ligand biased-behavior (Fig. 4). Interestingly, a low versus high $\mathrm{G}$ protein stoichiometric-dependence of ligands could not be appreciated from a downstream $\mathrm{G}$ protein effector, i.e. the 
a
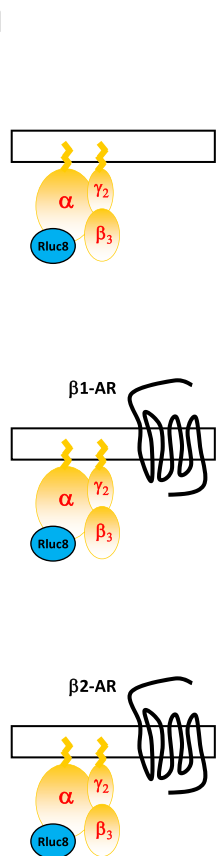

b
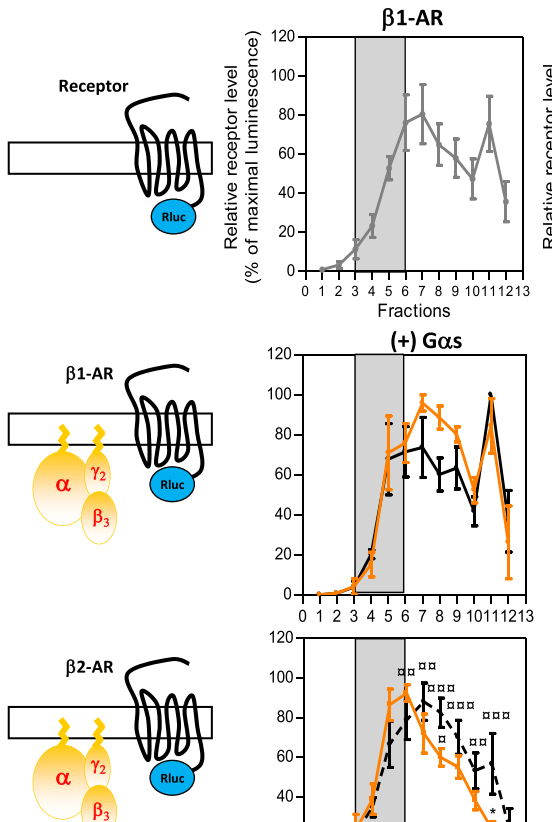

$(+)$ Gas
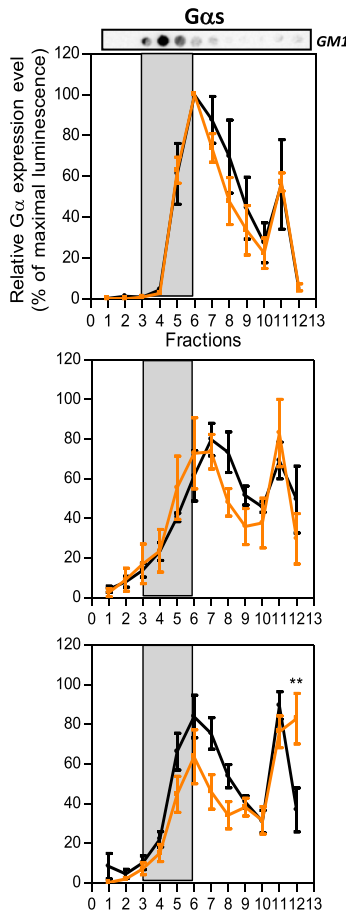

B1-AR
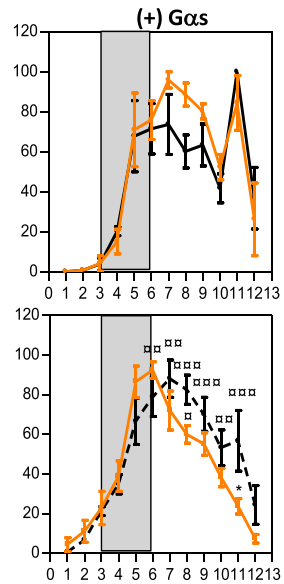
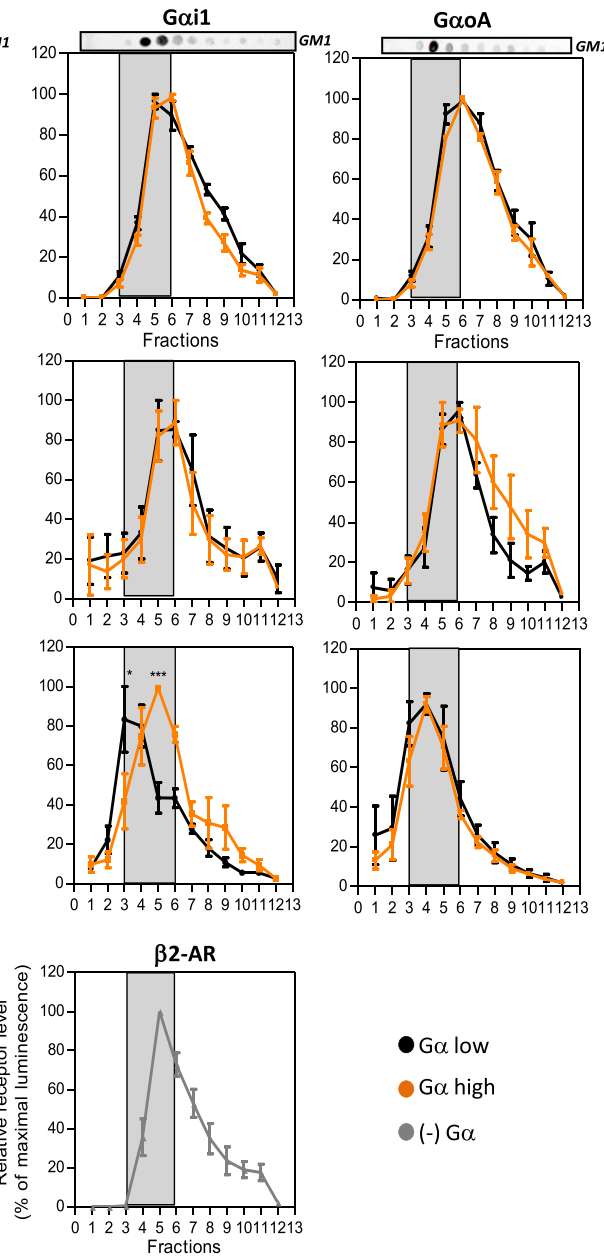

(+) Gai1
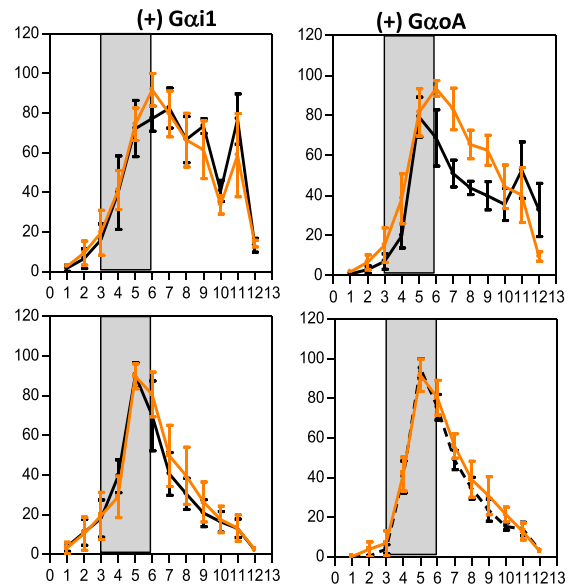

Figure 6. Go expression level influences $\mathrm{G} \alpha$ subunit and $\beta$-AR receptor compartmentalization in cholesterolenriched membranes. Detergent-resistant-membranes were purified using a triton X-100 lysis method followed by a separation on sucrose gradient from (a) HEK293T cells co-expressing high (orange) and low (black) expression levels of Gos-Rluc8 (left panel), Gail-Rluc8 (middle panel) or GooA-Rluc8 (right panel) along with $G \beta 3$ and $G \gamma 2$ subunits in the presence or not (upper panels) of untagged $\beta$-AR receptors, (b) HEK293T cells co-expressing $\beta 1$-AR-Rluc or $\beta 2$-AR-Rluc alone (upper panels) or in the presence of high (orange) or low

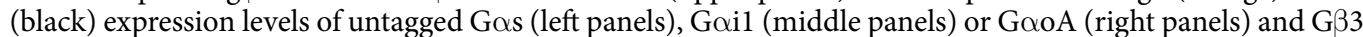
and $\mathrm{G} \gamma 2$ subunits. Relative receptor or $\mathrm{G} \alpha$ subunit expression levels were quantified in each sucrose fractions by recording of the total luminescence. Results are expressed as the percentage of the maximal luminescence measured from all fractions in each experiment. Grey boxes highlight raft nano-domains enriched fractions, identified by detection of the GM1 protein (upper dot plot). Data represent the mean \pm s.e.m. of at least three independent experiments. The statistical significance of the difference in membrane distribution in low versus high conditions $(*)$ or versus receptor alone $(a)$ was assessed using two-way ANOVA followed by a Bonferroni posttest $(* P<0.05, * * P<0.01, * * * P<0.001)$. 
adenylyl cyclase activity promoted by Gs (Supplementary Fig. 4), most probably due to the amplification and saturation of the second messenger production. Such dichotomy could also rely on experimental differences as the BRET assay specifically measured the $G \alpha_{X} \beta 3 \gamma 2$ protein complex activity while cAMP production is more global and most likely relies on a mixture of $\beta$-AR signals emanating from different $\mathrm{G}$ protein isoform combinations.

Indeed, the GPCR activation theory mainly assumes a free receptor/G protein/effector system in a fluid mosaic model where the membrane is considered a homogeneous lipid medium. However, biological membranes are much more complex and harbor compartments with different component partitions, thus introducing the notion of a spatial signaling constraint. GPCRs and G proteins, as inserted membrane proteins, do not escape this rule. In a mammalian cell, each of them are subjected to different lipid post-translational modifications ${ }^{36,39}$ that dictate particular membrane subdomain localizations and activities. In this study, we demonstrated that the

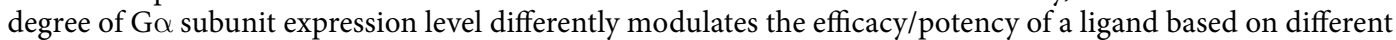
partitioning of both the $G$ protein and the receptor, at least in part, in raft-enriched domains. In agreement with microdomain-dependent ligand efficacy, we found that the $G$ protein level stabilizes different conformations of the receptor-G $\alpha$ complex. This observation completely reconciles the ligand-biased-behavior that until now was inferred from the stabilization of different receptor conformations ${ }^{11}$. Several studies have already reported the different influences of the lipid membrane composition on receptor pharmacology ${ }^{33,40}$ and specifically cholesterol enriched in raft domains was recently identified as a key trigger that can directly bind and activate GPCRs

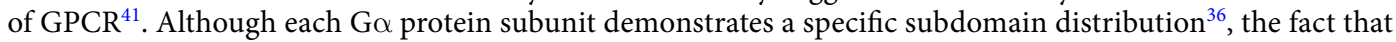

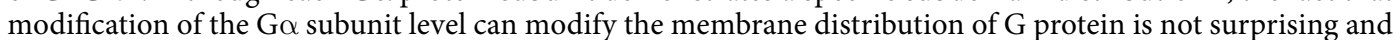
could be easily reconciled with its overexpression resulting in saturation of certain cell compartments with consequent occupation of new ones. Accordingly, endogenous adenylyl cyclase 6 was shown to be localized primarily in caveolae-rich domains in rat aortic smooth muscle cells while overexpressed AC6 was localized only in non-caveolae domains ${ }^{42}$. More outstanding finding is the impact of the Go expression on the receptor partitioning at resting state since receptor shuttling in different membrane domains has been mostly reported upon agonist stimulation ${ }^{43}, 44$. Overall, this result could indeed refer to the existence of a preformed receptor-G protein complex that several groups have already described ${ }^{45-48}$. In this model, the biochemical properties of both the receptor and the $\mathrm{G}$ protein in the complex could differ from each individual protein taken separately and thus dictate specific trafficking-partitioning properties depending on the $G$ protein and the receptor species as shown by our results. In line with this hypothesis, despite the presence of palmitoylation lipid modifications, some proteins like CD4 do not necessarily require palmitoylation for lipid raft localization ${ }^{49}$. Finally, in agreement with this model, Scarlata et al. previously suggested a role for $\mathrm{Gq}$ in directing the bradykinin type 2 receptor into caveolae domains ${ }^{50}$.

In addition to the $G$ protein stoichiometry parameter, in this study we have also confirmed the influence of the $\mathrm{G}$ protein isoform on the functional selectivity of ligands, as recently outlined ${ }^{12,13}$. From this standpoint, an important finding is that the three $\beta$-AR agonists, ISO, NE and EPI, behaved as biased agonists relative to each other, as reflected by their differences in potencies/efficacies on the activation of the three G proteins tested, namely Gos, GooA and Goil (Fig. 4). This result corroborates that of Lohse's group who demonstrated that these agonists stabilize different $\beta 2$-AR conformations ${ }^{51}$. Hence, ISO, NE and EPI most probably promote selective signaling pathways and cellular responses at $\beta$-ARs with different physio/pathophysiological responses. These data should have major impact on the $\beta$-adrenergic field in which ISO is usually taken as a more stable surrogate agonist for natural NE /EPI especially when exploring the physio/pathophysiology of $\beta$-AR-dependent cardiovascular regulation. It therefore follows that previous conclusions obtained with ISO to make an assumption on NE or EPI effects in physio/pathophysiology should be reconsidered.

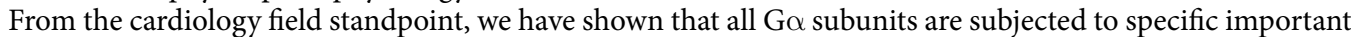
gene expression regulation in cardiomyocytes under pathological situation such as barometric-induced failure.

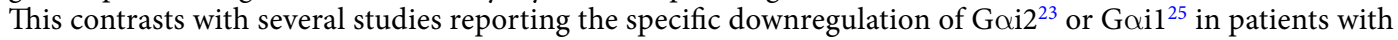
heart failure. Although this discrepancy could rely on the specificity of the phenotype, it could also result from an experimental issue since these studies examined gene expression in whole heart samples harboring different cardiac cell types and not in isolated cardiomyocytes. It will thus be interesting in the future to test whether different cardiac diseases dictate a specific fingerprint of Go gene expression. Another interesting finding from our study is the specific downregulation of Goil in the context of barometric cardiac stress, which correlated in heterologous system with a gain in its coupling to the $\beta$-AR and which could therefore account for the Gs to Gi signaling switch of the $\beta 2$-AR that has been reported in pathological situation $s^{52}$. Finally, given that modification of $\beta$-AR signaling has clearly been established as a molecular contributor to the evolution of heart failure, changes in the $G$ protein and the receptor ratio could participate in the atypical pharmacology of $\beta$-AR agonists and the onset and/or worsening of the disease. Although this assumption could also be extended to $\beta$-antagonists, an interesting finding from our study is the lower dependence to the $\mathrm{G \alpha}$ stoichiometry of metoprolol and bisoprolol, two $\beta$-blockers currently used in the management of chronic heart failure improving symptoms and reducing mortality. This is an intriguing finding when considering our initial results showing high fluctuations in G $\alpha$ gene expression levels in failing cardiomyocytes. Indeed, on one hand, preserved efficacy of $\beta$-blockers across different G $\alpha$ expression levels could account, at least in part, for their clinical efficacy but also for the lack of clear clinical advantage of one drug over the other ${ }^{53}$. On the other hand, the high variability of responses to $\beta$-blockers compared to $\beta$-agonists in our experiments, especially in the low Goil and GooA level range, could also refer to the still unpredictable $30 \%$ of non-responders to $\beta$-blockers in clinical trials. Altogether, these results further reinforce the recent notion of dynamic bias and its implication for GPCR drug $d$ iscovery ${ }^{54}$.

In summary, the present study highlights the importance of $G$ protein stoichiometry as an important contributor to the bias of ligands. Thus, the biased activity of ligands should be considered when comparing cells with similar $\mathrm{G}$ protein repertoires. However, the stoichiometry of the other signaling partners will most probably affect the bias of ligands as well. This truly questions the relevance of bias signaling analysis/quantification in 
recombinant systems that have been used as standard up to now when comparing different signaling pathways with variable manipulation of receptor and effector expression levels ( $G$ protein, $\beta$-arrestin, GRK...) ${ }^{19}$. From an industrial standpoint, our results could help fine tuning the assays used in HTS programs to increase the yield of positive hits for biased ligand identification. However, even with the most biased hit ligand, its exact bias translation in in vivo systems with varying cell types and signaling components will still remain a tall order and will not necessarily predict its therapeutic efficacy. Thus, the in vitro identification of biased ligands does not have to ring as a predictive therapeutic value but more as a yield component and all molecule hits obtained from this in vitro screen will have to be systematically tested in vivo without a priori and without shortlisting.

\section{Methods}

Live animals. Experimental animal protocols were carried out in two month old male C57BL/6 mice in accordance with the French regulation guidelines for animal experimentation and were approved by the CEEA122 ethical committee.

Materials. (-)-Isoproterenol hydrochloride (ISO), (-)-norepinephrine $(+)$ bitartrate salt monohydrate (NE), (-)-epinephrine (EPI), and angiotensine II (AngII) were purchased from Sigma-Aldrich (St. Louis, MO, USA) while coelenterazine $400 a$ and coelenterazine $h$ were purchased from Interchim.

Quantification of $\mathrm{G} \alpha$ subunits gene expression. Pathological cardiac hypertrophy was induced in 6-week-old male C57BL/6 J mice by transversal aortic banding (TAC). Animals were analyzed 15 days after TAC and their cardiac characterization was previously reported ${ }^{28}$. Fifteen days after TAC, cardiomyocytes were then isolated and total RNA isolation and real-time quantitative RT-PCR were conducted as previously described ${ }^{28}$.

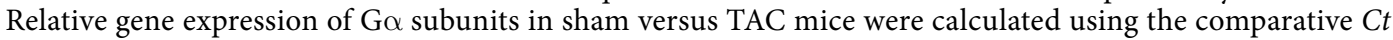
method after quantitative PCR performed using the Fluidigm Biomark HD nano-scale platform. Genes encoding the Glyceraldehyde-3-phosphate dehydrogenase (GAPDH) and the Hypoxanthine-guanine phosphoribos-

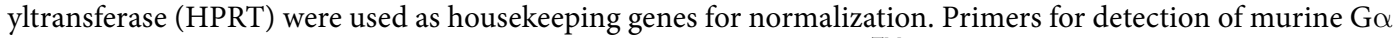
subunits genes were provided and validated by Fluidigm (DELTAgene ${ }^{\mathrm{TM}}$ assays).

cDNA expression vectors. Plasmids encoding HA-AT ${ }_{1 A}-\mathrm{R}, \mathrm{HA}-\beta 2-\mathrm{AR}, \beta 1$-AR-GFP10, $\beta 2$-AR-Rluc,

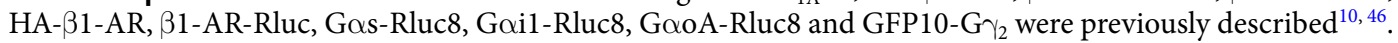
G $\beta 3$ encoding vector was obtained from the Missouri S\&T cDNA Resource Center.

Cell culture and transfection. Human embryonic kidney 293 cells (HEK293T) cells were cultured in DMEM Glutamax supplemented with $10 \%$ (v/v) FBS and 100 units $/ \mathrm{ml}$ penicillin/streptomycin at $37^{\circ} \mathrm{C}$ in a humidified atmosphere at $5 \%$ CO2. Transient transfections were performed $24 \mathrm{~h}$ after cell seeding using polyethylenimine (PEI, Polysciences Inc.).

Bioluminescence resonance energy transfer (BRET) measurements. Receptor and G proteins subunit-encoding vectors were transiently transfected into HEK293T cells as indicated in the figure legends. Forty-eight hours after transfection, cells were washed with PBS, detached in PBS/5 mM EDTA and resuspended in $\mathrm{PBS} / 0.1 \%(\mathrm{w} / \mathrm{v})$ glucose at room temperature. Cells were then distributed ( $80 \mu \mathrm{g}$ of protein per well) into a 96-well microplate (Wallac, PerkinElmer Life and Analytical Sciences) and incubated in the presence of the different ligands for $1 \mathrm{~min}$. BRET ${ }^{2}$ between Rluc8 and GFP10 was measured after the addition of the Rluc8 substrate coelenterazine $400 a(5 \mu \mathrm{M}$, Interchim). BRET readings were collected using a modified Infinite F500 (Tecan Group Ltd). The BRET ${ }^{2}$ signal was calculated by the ratio of GFP10 emission ( $\left.510-540 \mathrm{~nm}\right)$ to that of RLuc/Rluc8 $(370-450 \mathrm{~nm})$.

Biochemical purification of cholesterol-enriched microdomains. Receptor and G proteins were transiently transfected in HEK293T cells as indicated in the figure legends. Detergent-resistant membrane microdomains isolation was conducted 48 hours after transfection as previously described ${ }^{34}$. Briefly, cells were washed, detached in cold PBS and solubilized in ice-cold lysis buffer (Tris $25 \mathrm{mM} \mathrm{pH} \mathrm{7.4;} \mathrm{NaCl} 140$ mM; EDTA $2 \mathrm{mM}$ ) containing $1 \%$ Triton $\times 100(\mathrm{v} / \mathrm{v})($ Sigma) and a protease inhibitor cocktail (Complete mini; Roche, Bale, Switzerland). Lysates were then homogenized and centrifuged $10 \mathrm{~min}$ at $860 \mathrm{~g}$ at $4{ }^{\circ} \mathrm{C}$. Supernatants were collected and protein content was evaluated using the BioRad $\mathrm{DC}^{\mathrm{TM}}$ protein assay. An equal amount of proteins for each individual transfection was then adjusted to $60 \%$ sucrose and placed at the bottom of an Ultra-Clear centrifuge tube (Beckman instruments), and overlaid with a 5-35\% discontinuous sucrose gradient prepared in the lysis buffer (without triton). Gradients were centrifuged at $39000 \mathrm{rpm}$ for $18 \mathrm{~h}$ at $4{ }^{\circ} \mathrm{C}$ without breakdown in a SW41 rotor (Beckman L70 Ultracentrifuge). Then, twelve $1 \mathrm{ml}$ fractions were collected from the top of the gradients. Total luminescence in each fraction $(180 \mu \mathrm{l})$ was measured in a 96-well microplate following addition of $5 \mu \mathrm{M}$ of the luciferase substrate, coelenterazine $h$, using a luminometer Mithras LB 940 (Berthold technologies, Germany). GM1 gangliosides, a marker of the raft microdomains, was detected in each fraction by dot plot using peroxydase-coupled cholera toxin subunit B conjugates (Molecular probes). Sucrose density was controlled by refractometry.

Quantification of cell surface receptors by ELISA. HA-tagged receptors and G proteins were transiently transfected in HEK293T cells as indicated in the figure legends. Twenty-four hours after transfection, cells were splited in 24-well plates precoated with poly-L-lysine. The next day, cells were fixed in $4 \%$ paraformaldehyde, saturated with PBS containing $1 \%$ bovine serum albumin and incubated with the primary anti-HA antibody 
(clone 16B12; Covance) and then with the HRP-labeled secondary antibody (Sigma, St. Louis, MO, USA). After washing, cells were incubated with HRP substrate TMB (3,39,5,59-tetramethylbenzidine; BD Biosciences). The reaction was stopped with $\mathrm{HCl} 1 \mathrm{~N}$ and the plates were read at $450 \mathrm{~nm}$ in a microplate reader (Varioscan Flash, Thermo Electron). The $570 \mathrm{~nm}$ optic density (background) was subtracted according to the manufacturer.

Western blot analysis. Receptor and G proteins were transiently transfected in HEK293T cells as indicated

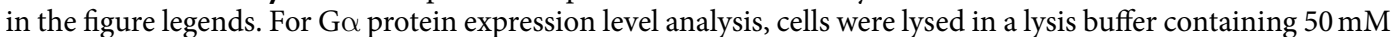
Tris $\mathrm{pH} 7.4,150 \mathrm{mM} \mathrm{NaCl}, 1 \mathrm{mM}$ EDTA, $1 \%$ triton, $\mathrm{H} 2 \mathrm{O}$ and cocktail protease inhibitors (ROCHE). Cells lysates $(50 \mu \mathrm{g})$ were subjected to SDS-PAGE electrophoresis under reducing conditions, transferred onto PVDF membranes (Millipore), and analyzed by immunoblotting according to standard protocols using anti-Gos polyclonal

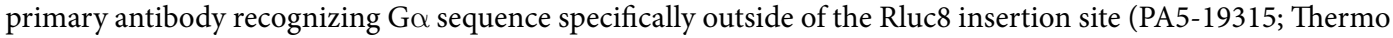
fisher Scientific), and an anti-GAPDH monoclonal antibody (sc-47724; Santa Cruz Biotechnology). Rabbit anti-Goat and Sheep anti-Mouse HRP-labeled secondary antibodies were from Thermo fisher Scientific (31402) and GE Healthcare (NA931V) respectively.

cAMP quantification. Quantification of cAMP levels was performed using the HTRF assay (Homogeneous Time-Resolved Fluorescence): dynamic2 cAMP kit (Cisbio, Bedford, USA), based on a competitive immunoassay using Lumi4-TbTM cryptate-labeled antibodies anti-cAMP and d2-labeled cAMP. For that purpose, HEK293T cells were transiently transfected as indicated in the figure legends and $48 \mathrm{~h}$ post-transfection, cells were washed and resuspended in PBS/5 mM Glucose/2 mM IBMX. Then, 10,000 cells/well (384 wells-plate) were plated and stimulated for $1 \mathrm{~h}$ at room temperature with increasing ligand concentrations in a final volume of $10 \mu \mathrm{L}$. Cells were then lysed using $5 \mu \mathrm{L}$ of lysis buffer containing d2-labeled cAMP and $5 \mu \mathrm{L}$ of Lumi4-TbTM cryptate-labeled anti-cAMP. The signal was measured after $1 \mathrm{~h}$ room temperature incubation using a modified Infinite F500 (Tecan Group Ltd). The RET signal was calculated by the ratio of d2-cAMP/Lumi4-TbTM ( $665 \mathrm{~nm} / 620 \mathrm{~nm})$, the specific signal being inversely proportional to the concentration of cAMP in the sample. For each experiment, a calibration curve was established with cAMP standards allowing the quantification of cAMP levels by linear regression.

Data and statistical analysis. Statistical analysis of genomic changes between sham and TAC cardiomyocytes and BRET signal modulations were carried out using GraphPad Prism 4 software (GraphPad Software Inc., San Diego, CA). Statistical tests used are indicated in the figure legends. Values of $P<0.05$ were considered statistically significant.

\section{References}

1. Kenakin, T. Functional selectivity and biased receptor signaling. J Pharmacol Exp Ther 336, 296-302 (2011).

2. Kingwell, K. Pioneering biased ligand offers efficacy with reduced on-target toxicity. Nat Rev Drug Discov 14, 809-810 (2015).

3. Patel, C. B., Noor, N. \& Rockman, H. A. Functional selectivity in adrenergic and angiotensin signaling systems. Mol Pharmacol 78, 983-992 (2010)

4. Kubon, C. et al. The role of beta-blockers in the treatment of chronic heart failure. Trends Pharmacol Sci 32, 206-212, doi:10.1016/j. tips.2011.01.006 (2011)

5. Poole-Wilson, P. A. et al. Comparison of carvedilol and metoprolol on clinical outcomes in patients with chronic heart failure in the Carvedilol Or Metoprolol European Trial (COMET): randomised controlled trial. Lancet 362, 7-13, doi:10.1016/S01406736(03)13800-7 (2003).

6. Thanawala, V. J. et al. Ligand bias prevents class equality among beta-blockers. Curr Opin Pharmacol 16, 50-57 (2014).

7. Masri, B. et al. Antagonism of dopamine D2 receptor/beta-arrestin 2 interaction is a common property of clinically effective antipsychotics. Proc Natl Acad Sci USA 105, 13656-13661, doi:10.1073/pnas.0803522105 (2008).

8. Allen, J. A. et al. Discovery of beta-arrestin-biased dopamine D2 ligands for probing signal transduction pathways essential for antipsychotic efficacy. Proc Natl Acad Sci USA 108, 18488-18493, doi:10.1073/pnas.1104807108 (2011).

9. Wei, H. et al. Independent beta-arrestin 2 and G protein-mediated pathways for angiotensin II activation of extracellular signalregulated kinases 1 and 2. Proc Natl Acad Sci USA 100, 10782-10787, doi:10.1073/pnas.1834556100 (2003).

10. Sauliere, A. et al. Deciphering biased-agonism complexity reveals a new active AT1 receptor entity. Nat Chem Biol 8, 622-630 (2012).

11. Shukla, A. K. et al. Distinct conformational changes in beta-arrestin report biased agonism at seven-transmembrane receptors. Proc Natl Acad Sci USA 105, 9988-9993, doi:10.1073/pnas.0804246105 (2008).

12. Klein Herenbrink, C. et al. The role of kinetic context in apparent biased agonism at GPCRs. Nat Commun 7, 10842, doi:10.1038/ ncomms10842 (2016)

13. Masuho, I. et al. Distinct profiles of functional discrimination among G proteins determine the actions of $G$ protein-coupled receptors. Sci Signal 8, ra123, doi:10.1126/scisignal.aab4068 (2015).

14. Kenakin, T. Differences between natural and recombinant $G$ protein-coupled receptor systems with varying receptor/G protein stoichiometry. Trends Pharmacol Sci 18, 456-464 (1997).

15. Kenakin, T. Efficacy at G-protein-coupled receptors. Nat Rev Drug Discov 1, 103-110, doi:10.1038/nrd722 (2002).

16. Kenakin, T. Gaddum Memorial Lecture 2014: receptors as an evolving concept: from switches to biased microprocessors. $\mathrm{Br} J$ Pharmacol 172, 4238-4253 (2015).

17. Kenakin, T. P. Synoptic pharmacology: Detecting and assessing the pharmacological significance of ligands for orphan receptors. Pharmacol Res 114, 284-290 (2016).

18. Watson, C. et al. The use of stimulus-biased assay systems to detect agonist-specific receptor active states: implications for the trafficking of receptor stimulus by agonists. Mol Pharmacol 58, 1230-1238 (2000).

19. Galandrin, S., Onfroy, L., Poirot, M. C., Senard, J. M. \& Gales, C. Delineating biased ligand efficacy at 7TM receptors from an experimental perspective. Int J Biochem Cell Biol 77, 251-263 (2016).

20. Doi, M. et al. Gpr176 is a Gz-linked orphan G-protein-coupled receptor that sets the pace of circadian behaviour. Nat Commun 7 , 10583 (2016).

21. Grant, K. R., Harnett, W., Milligan, G. \& Harnett, M. M. Differential G-protein expression during B-and T-cell development. Immunology 90, 564-571 (1997).

22. Chang, G. W. et al. CD312, the human adhesion-GPCR EMR2, is differentially expressed during differentiation, maturation, and activation of myeloid cells. Biochem Biophys Res Commun 353, 133-138 (2007).

23. Eschenhagen, T. et al. Increased messenger RNA level of the inhibitory G protein alpha subunit Gi alpha- 2 in human end-stage heart failure. Circ Res 70, 688-696 (1992). 
24. Ishikawa, Y. et al. Downregulation of adenylylcyclase types V and VI mRNA levels in pacing-induced heart failure in dogs. J Clin Invest 93, 2224-2229 (1994).

25. Longabaugh, J. P., Vatner, D. E., Vatner, S. F. \& Homcy, C. J. Decreased stimulatory guanosine triphosphate binding protein in dogs with pressure-overload left ventricular failure. J Clin Invest 81, 420-424, doi:10.1172/JCI113335 (1988).

26. Yajima, I. et al. Reduced GNG2 expression levels in mouse malignant melanomas and human melanoma cell lines. Am J Cancer Res 2, 322-329 (2012).

27. Corvol, J. C. et al. Persistent increase in olfactory type G-protein alpha subunit levels may underlie D1 receptor functional hypersensitivity in Parkinson disease. J Neurosci 24, 7007-7014, doi:10.1523/JNEUROSCI.0676-04.2004 (2004).

28. Genet, G. et al. Ephrin-B1 is a novel specific component of the lateral membrane of the cardiomyocyte and is essential for the stability of cardiac tissue architecture cohesion. Circ Res 110, 688-700 (2012).

29. Bellot, M. et al. Dual agonist occupancy of AT1-R-alpha2C-AR heterodimers results in atypical Gs-PKA signaling. Nat Chem Biol 11, 271-279 (2015).

30. Rybin, V. O. \& Steinberg, S. F. G protein betagamma dimer expression in cardiomyocytes: developmental acquisition of Gbeta3. Biochem Biophys Res Commun 368, 408-413, doi:10.1016/j.bbrc.2008.01.100 (2008).

31. Urizar, E. et al. CODA-RET reveals functional selectivity as a result of GPCR heteromerization. Nat Chem Biol 7, 624-630 (2011).

32. M’Kadmi, C. et al. Agonism, Antagonism, and Inverse Agonism Bias at the Ghrelin Receptor Signaling. J Biol Chem 290, 27021-27039 (2015).

33. Dawaliby, R. et al. Allosteric regulation of G protein-coupled receptor activity by phospholipids. Nat Chem Biol 12, 35-39 (2016).

34. Pontier, S. M. et al. Cholesterol-dependent separation of the beta2-adrenergic receptor from its partners determines signaling efficacy: insight into nanoscale organization of signal transduction. J Biol Chem 283, 24659-24672 (2008).

35. Becher, A., White, J. H. \& Mcllhinney, R. A. The gamma-aminobutyric acid receptor B, but not the metabotropic glutamate receptor type-1, associates with lipid rafts in the rat cerebellum. J Neurochem 79, 787-795 (2001).

36. Wedegaertner, P. B., Wilson, P. T. \& Bourne, H. R. Lipid modifications of trimeric G proteins. J Biol Chem 270, 503-506 (1995).

37. Azzi, M. et al. Allosteric effects of $\mathrm{G}$ protein overexpression on the binding of beta-adrenergic ligands with distinct inverse efficacies. Mol Pharmacol 60, 999-1007 (2001).

38. Yao, X. J. et al. The effect of ligand efficacy on the formation and stability of a GPCR-G protein complex. Proc Natl Acad Sci USA 106, 9501-9506 (2009).

39. Qanbar, R. \& Bouvier, M. Role of palmitoylation/depalmitoylation reactions in G-protein-coupled receptor function. Pharmacol Ther 97, 1-33 (2003).

40. Zocher, M., Zhang, C., Rasmussen, S. G., Kobilka, B. K. \& Muller, D. J. Cholesterol increases kinetic, energetic, and mechanical stability of the human beta2-adrenergic receptor. Proc Natl Acad Sci USA 109, E3463-3472 (2012).

41. Huang, P. et al. Cellular Cholesterol Directly Activates Smoothened in Hedgehog Signaling. Cell 166, 1176-1187 e1114 (2016).

42. Ostrom, R. S. et al. Localization of adenylyl cyclase isoforms and G protein-coupled receptors in vascular smooth muscle cells: expression in caveolin-rich and noncaveolin domains. Mol Pharmacol 62, 983-992 (2002).

43. Pike, L. J. Lipid rafts: bringing order to chaos. J Lipid Res 44, 655-667 (2003).

44. Rybin, V. O., Xu, X., Lisanti, M. P. \& Steinberg, S. F. Differential targeting of beta -adrenergic receptor subtypes and adenylyl cyclase to cardiomyocyte caveolae. A mechanism to functionally regulate the cAMP signaling pathway. J Biol Chem 275, 41447-41457 (2000).

45. Damian, M. et al. Ghrelin receptor conformational dynamics regulate the transition from a preassembled to an active receptor:Gq complex. Proc Natl Acad Sci USA 112, 1601-1606 (2015).

46. Gales, C. et al. Probing the activation-promoted structural rearrangements in preassembled receptor-G protein complexes. Nat Struct Mol Biol 13, 778-786 (2006).

47. Nobles, M., Benians, A. \& Tinker, A. Heterotrimeric G proteins precouple with G protein-coupled receptors in living cells. Proc Natl Acad Sci USA 102, 18706-18711, doi:10.1073/pnas.0504778102 (2005).

48. Qin, K., Dong, C., Wu, G. \& Lambert, N. A. Inactive-state preassembly of G(q)-coupled receptors and G(q) heterotrimers. Nat Chem Biol 7, 740-747, doi:10.1038/nchembio.642 (2011).

49. Popik, W. \& Alce, T. M. CD4 receptor localized to non-raft membrane microdomains supports HIV-1 entry. Identification of a novel raft localization marker in CD4. J Biol Chem 279, 704-712 (2004).

50. Calizo, R. C. \& Scarlata, S. A role for G-proteins in directing G-protein-coupled receptor-caveolae localization. Biochemistry 51, 9513-9523 (2012).

51. Reiner, S., Ambrosio, M., Hoffmann, C. \& Lohse, M. J. Differential signaling of the endogenous agonists at the beta2-adrenergic receptor. J Biol Chem 285, 36188-36198 (2010).

52. Gong, H. et al. Specific beta(2)AR blocker ICI 118,551 actively decreases contraction through a G(i)-coupled form of the beta(2)AR in myocytes from failing human heart. Circulation 105, 2497-2503 (2002).

53. Barrese, V. \& Taglialatela, M. New advances in beta-blocker therapy in heart failure. Front Physiol 4, 323 (2013)

54. Michel, M. C., Seifert, R. \& Bond, R. A. Dynamic bias and its implications for GPCR drug discovery. Nat Rev Drug Discov 13, 869 (2014)

\section{Acknowledgements}

J.M.S. and C.G. are supported by the Institut National de la santé et de la Recherche Médicale and by grant from the Fondation Bettencourt-Schueller (C.G.).

\section{Author Contributions}

L.O. designed and performed most of the experiments, analyzed, interpreted data and help to write the manuscript. S.G. conducted and analyzed cAMP production experiments. S.M.P. analyzed sucrose gradients, conceived some experiments and wrote some aspects of the manuscript. M.H.S. and D.N. assisted in experiments. J.M.S. assisted in data processing performed the statistical analysis and corrected the manuscript. C.G. conceived and supervised the project, performed data analysis and wrote the manuscript.

\section{Additional Information}

Supplementary information accompanies this paper at doi:10.1038/s41598-017-07392-5

Competing Interests: The authors declare that they have no competing interests.

Publisher's note: Springer Nature remains neutral with regard to jurisdictional claims in published maps and institutional affiliations. 
(i) Open Access This article is licensed under a Creative Commons Attribution 4.0 International License, which permits use, sharing, adaptation, distribution and reproduction in any medium or format, as long as you give appropriate credit to the original author(s) and the source, provide a link to the Creative Commons license, and indicate if changes were made. The images or other third party material in this article are included in the article's Creative Commons license, unless indicated otherwise in a credit line to the material. If material is not included in the article's Creative Commons license and your intended use is not permitted by statutory regulation or exceeds the permitted use, you will need to obtain permission directly from the copyright holder. To view a copy of this license, visit http://creativecommons.org/licenses/by/4.0/.

(C) The Author(s) 2017 